\section{OP0333 DISCOVERY AND VALIDATION OF NOVEL AUTOANTIGENS IN SJÖGREN'S SYNDROME WITH POTENTIAL FOR SUBGROUPING OF DISEASE}

P.K. Schulz-Knappe ${ }^{1}$, P. Budde ${ }^{1}$, H.-D. Zucht ${ }^{1}$, D. Wirtz ${ }^{1}$, K. Marquardt ${ }^{1}$, R. Thomas ${ }^{1}$, T. Witte ${ }^{2}$, M. Schneider ${ }^{3}$, K. Sivils ${ }^{4}$, A. Rasmussen ${ }^{5} .{ }^{1}$ Protagen, Dortmund; ${ }^{2}$ Dept. of Clinical Immunology and Rheumatology, Hannover Medical School, Hannover; ${ }^{3}$ Dept. of Rheumatology, Heinrich Heine University, Düsseldorf, Germany; ${ }^{4}$ Oklahoma Sjogren's Syndrome Center of Research Translation; ${ }^{5}$ Oklahoma Medical Research Foundation, Oklahoma, United States

Background: Primary Sjögren's syndrome (pSS) is a common autoimmune disease with exocrine gland dysfunction and multi-organ involvement. With the growing interest in conducting clinical trials in pSS, there is a need for new biomarkers that can be used to diagnose pSS, identify clinical subsets of pSS, predict treatment outcome and assessment of disease activity. Activation of B-cells and dysregulation of the cytokine network plays a critical role in the pathophysiology of pSS. In the exocrine glands, elevated levels of cytokines, such as type I interferon (IFN), tumor necrosis factor alpha (TNF), interleukin 12 (IL-12) and $B$ cell activating factor (BAFF) can be found. Dysregulated pathways of the innate and adaptive immune system lead to loss of tolerance and the production of organ-specific and non-specific autoantibodies. Current diagnostic criteria for pSS utilize autoantibodies directed to nuclear antigens (ANA), especially to SS-A/Ro (TRIM21, TROVE2) and La (SSB), but those are not specific, and can be identified as well in SLE and even in healthy volunteers. Several studies have shown that not all patients with pSS are tested positive for Ro and La autoantibodies, but suggested the existence of additional autoantibodies in pSS. This autoantibody burden is not well understood for the importance of disease progression, for its role in patient segmentation, or for response to treatments.

Objectives: The discovery of novel autoantigens may provide a deeper understanding of mechanisms of actions for pSS drugs, and may be useful to stratify patients.

Methods: The autoantibody reactivity pattern of pSS serum patients was analyzed using a Luminex bead-based antigen array (SeroTag) and 1,600 selected human protein antigens from our hPEX protein library of 8,000 recombinant proteins (1). We screened over 2,000 serum samples from patients with autoimmune diseases as active controls targeting Sjögren's Syndrome $(n=70)$, SLE $(n=>500)$, SSc $(n=>250), R A(n=>500)$, and over 1,000 healthy individuals to confirm known and to discover novel autoantibodies. In a validation study, novel biomarker candidate antigens were evaluated using a cohort of 350 Patients with Sjögren's Syndrome. Results: Apart from clear confirmation the known benchmark autoantigens known for many years we have discovered a small set of additional, novel autoantibodies, which were detected in frequencies of 8 to $>20 \%$ in pSS. Accumulation of autoantibody reactivities allows for a first subgroup definition of Sjögren's, and for clear segregation of SjS/SLE overlap syndrome patients.

Conclusions: A set of novel autoantigens for diagnosis and subgroup definition in Sjögren's syndrome was discovered by high content screening using a Luminex bead-based array platform. Validation in additional, large patient cohorts is ongoing.

References:

[1] Budde P et al. (2016). Lupus. (8):812-22.

Disclosure of Interest: P. Schulz-Knappe Shareholder of: Protagen AG, P. Budde Employee of: Protagen AG, H.-D. Zucht: None declared, D. Wirtz Employee of: Protagen AG, K. Marquardt Employee of: Protagen AG, R. Thomas: None declared, T. Witte: None declared, M. Schneider Consultant for: Protagen AG, K. Sivils: None declared, A. Rasmussen: None declared

DOI: 10.1136/annrheumdis-2017-eular.5725

\section{SATURDAY, 17 JUNE 2017 Optimizing treatment for osteoarthritis: take the phenotype in account "one size does not fit all"}

\section{OP0334 PREDICTORS AND MRI-DETECTED STRUCTURAL PATHOLOGY WITH TRAJECTORIES OF KNEE PAIN SEVERITY: A 10.7-YEAR PROSPECTIVE STUDY}

F. Pan ${ }^{1}$, J. Tian ${ }^{2}$, D. Aitken ${ }^{1}$, F. Cicuttini ${ }^{3}$, C. Ding ${ }^{1}$, G. Jones ${ }^{1}$ ${ }^{1}$ Musculoskeletal Unit; ${ }^{2}$ Public Health, Menzies Institute for Medical Research, University of Tasmania: ${ }^{3}$ Department of Epidemiology and Preventive Medicine, Monash University Medical School, Hobart, Australia

Background: Knee pain is the most common manifestation of knee osteoarthritis $(\mathrm{OA})$ and is generally thought to get worse with time. Risk factors for knee pain have been extensively investigated; however, whether the risk factors are associated with a specific pain trajectory has not yet been comprehensively explored. Furthermore, knee structural pathology on MRI, such as bone marrow lesions (BMLs), effusion-synovitis and cartilage defects, are thought to be the origin of knee pain. However, its underlying mechanisms remain to be elucidated and hampered by a large individual variation of pain course.

Objectives: To identify distinct trajectories of knee pain over 10.7 years in an older population, to describe risk factors with identified trajectories, and to explore MRI-detected structural pathology with the trajectories.
Methods: 1,099 participants (mean age 63 years) from a population-based cohort study were recruited at baseline. 875,768 and 563 participants attended years 2.6, 5.1 and 10.7 follow-up, respectively. Demographic, psychological, lifestyle and comorbidities data were obtained at baseline. Knee pain was assessed using Western Ontario and McMaster Universities Osteoarthritis Index (WOMAC) at each time-point. T1-weighted or T2-weighted MRI of the right knee was performed to measure knee structural pathology. Knee radiographic OA was assessed by X-ray at baseline. Group-based trajectory modelling was applied to identify pain trajectories. Multi-nominal logistic regression was used for the analyses with adjustment for potential confounders.

Results: 1,099 participants were included for the identification of pain trajectories and three distinct pain trajectories were defined. Participants in Group 1 ("Mild pain" $n=568,51.7 \%$ ) had relatively stable mild pain over time. Participants in Group 2 ("Moderate pain", $n=366,33.2 \%$ ) had moderate pain over time. Participants in Group 3 ("Severe pain", n=165, 15.1\%) developed or displayed fluctuating severe pain over time. Compared with the "Mild pain", higher BMI, emotional problems, and musculoskeletal diseases were significantly associated with both "Moderate pain" and "Severe pain" trajectories. Also, younger age, lower education level and unemployment status were associated with "Severe pain" trajectory. The presence of cartilage defects and BMLs were associated with increased risk of "Moderate pain" and "Severe pain" trajectories before or after adjustment for potential confounders. Effusion-synovitis was not statistically associated with "Moderate pain" $(P=0.082)$, but associated with "Severe pain" trajectory. Furthermore, a dose-response relationship was observed between number of knee structural abnormalities, and "Moderate pain" and "Severe pain" trajectories (both $\mathrm{P}$ for trend $<0.001$ ).

Conclusions: This is the first long-term study to identify pain trajectories and their risk factors, and explore the associations between structural pathology and pain trajectories. Three distinct pain trajectory groups were identified, suggesting that homogeneous subgroups exist and follow a specific trajectory over time despite large individual variation of pain course. Significant associations between structural pathology and pain trajectories suggest that peripheral stimuli may play a role in the development and maintenance of pain severity.

Disclosure of Interest: None declared

DOI: 10.1136/annrheumdis-2017-eular.1412

\section{OP0335 EXPLORING ASSOCIATIONS BETWEEN HISTOLOGICALLY ASSESSED INFLAMMATION AND PAIN AND FATIGUE IN KNEE OSTEOARTHRITIS}

M.J. Minten ${ }^{1}$, A. Blom ${ }^{2}$, G.F. Snijders ${ }^{2}$, F.H. van den Hoogen ${ }^{2,3}$, A.A. den Broeder ${ }^{2,3}$, P. van der Kraan ${ }^{2}$, C.H. van den Ende ${ }^{1,2}$. ${ }^{1}$ Dept. of Research, Sint Maartenskliniek; ${ }^{2}$ Dept. of Rheumatology, Radboud University medical center; ${ }^{3}$ Dept. of Rheumatology, Sint Maartenskliniek, Nijmegen, Netherlands

Background: Pain and fatigue are frequent symptoms of osteoarthritis (OA) However, the exact mechanism causing these symptoms remains unclear. Inflammation is important in OA pathophysiology. Associations between inflammatory aspects and clinical outcomes have been found. However, mainly (contrastenhanced) MRI was used to assess synovial inflammation in these studies. Using this method, inflammation is assessed in an indirect way (e.g. infrapatellar fat pad signal enhancement, synovial fluid effusion and thickening of synovia tissue). Conversely, histological assessment of synovial inflammation is regarded as the gold standard. Using this method, specific aspects of inflammation can be distinguished and rated for severity.

Objectives: To evaluate the associations of inflammation-related histological parameters of the synovium with pain and fatigue in knee OA patients.

Methods: Fifty-nine patients fulfilling ACR criteria for knee OA were recruited from two prospective studies ${ }^{1,2}$ and gave consent for synovial biopsy using a mini knee arthroscopy. Biopsies were taken from visually inflamed areas of the synovium. Tissue sections were histologically assessed for 1) number of synovial lining cells (absolute); 2) sub-synovial infiltration; 3) fibrin deposition; 4) vascularization; 5) fibrosis; and 6) perivascular edema (scores $0-3$ ). ${ }^{3}$ Average scores across sections were calculated for each parameter and for all parameters together. These data were combined with longitudinal clinical data from the prospective studies (WOMAC pain, SF36 vitality; $0=$ worst $-100=$ best). Associations between individual inflammatory features and clinical outcomes over time were assessed with mixed model analyses.

Results: Patient characteristics are shown in Table 1. Relatively mild levels of inflammation were found, in line with previous research (median $1.1[95 \% \mathrm{Cl}$ $0.9-1.6])^{3,4}$ Longitudinal data was available for 56 patients. Age, gender, BMI and time since symptom onset were no confounders. No significant associations were found between any histological parameter and pain or fatigue over time (Table 2).

Table 1. Patient characteristics

\begin{tabular}{lc}
\hline & Mean $(\mathrm{Sd})$ \\
\hline Male/Female & $28 / 31$ \\
Age (years) & $56.4(9.8)$ \\
Symptom duration (years) & $10.1(10.3)$ \\
BMI (kg/m $\mathrm{m}^{2}$ ) & $29.7(5.9)$ \\
Follow-up (years) & $1.2(0.8)$ \\
\hline
\end{tabular}

Conclusions: This is the first study to examine different parameters of histologi- 
Table 2. Longitudinal associations

\begin{tabular}{lccc}
\hline & Median [25-75\%] & Pain B [95\% Cl] & Fatigue B [95\% CI] \\
\hline Synovial lining cells & $2.4[1.5-3.2]]$ & $0.5[-2.9-3.9]$ & $-2.3[-5.6-1.0]$ \\
Infiltrate & $1.2[0.5-1.8]$ & $2.9[-4.3-10.1]$ & $-4.7[-11.6-2.3]$ \\
Fibrin & $0.0[0.0-0.4]$ & $-7.1[-17.7-3.4]$ & $-0.1[-10.1-10.0]$ \\
Vascularization & $1.6[1.0-2.0]$ & $-1.2[-8.3-5.9]$ & $-0.5[-7.2-6.3]$ \\
Fibrosis & $0.8[0.2-1.3]$ & $-3.3[-10.4-3.8]$ & $0.8[-6.3-7.9]$ \\
Edema & $0.6[0.2-1.3]$ & $1.5[-4.8-7.8]$ & $0.2[-6.0-6.4]$ \\
Average inflammation & $1.1[0.9-1.6]$ & $-0.3[-11.0-10.5]$ & $-5.3[-15.6-5.0]$ \\
\hline
\end{tabular}

cally assessed synovial inflammation in knee OA patients and their associations with clinical outcomes over time. We were unable to identify individual inflammatory aspects which associate with pain or fatigue. Additional research is required to identify the underlying mechanism for pain and fatigue in $O A$.

References:

[1] Ann Rheum Dis 2011 70:1191-6.

[2] Scand J Rheum 2011 40:225-31.

[3] Clin Rheum 2010 29:1185-90.

[4] Arthritis Rheum 2005 52:3492-501.

Disclosure of Interest: None declared

DOI: 10.1136/annrheumdis-2017-eular.1776

\section{SATURDAY, 17 JUNE 2017}

Targeting adipose tissue inflammation

\section{OP0336 ROLE OF SYSTEMIC INFLAMMATION ASSOCIATED WITH RHEUMATOID ARTHRITIS IN THE GLUCOSE AND LIPID METABOLISM: HUMANS, CIA MOUSE MODEL AND IN VITRO STUDIES}

1. Arias de la Rosa ${ }^{1}$, S. Rodriguez-Cuenca ${ }^{2}$, Y. Jimenez-Gomez ${ }^{1}$ P. Ruiz-Limon ${ }^{1}$, C. Perez-Sanchez ${ }^{1}$, M.C. Abalos ${ }^{1}$, I. Cecchi ${ }^{1}$, J. Calvo ${ }^{1}$, R. Guzman ${ }^{3}$, M. Malagon ${ }^{3}$, E. Collantes ${ }^{1}$, A. Vidal-Puig ${ }^{2}$, C. Lopez-Pedrera ${ }^{1}$, A. Escudero-Contreras ${ }^{1}$, N. Barbarroja ${ }^{1} .{ }^{1}$ Rheumatology Service, IMIBIC/Reina Sofia Hospital/University of Cordoba, Cordoba, Spain: ${ }^{2}$ Metabolic Research Laboratories, Wellcome Trust-MRC Institute of Metabolic Science, Addenbroke's Hospital, University of Cambridge, Cambridge, United Kingdom; ${ }^{3}$ Department of Cell Biology, Physiology, and Immunology, IMIBIC/Reina Sofia

Hospital/University of Cordoba, Cordoba, Spain

Background: Rheumatoid arthritis (RA) patients are at higher risk for insulin resistance $(\mathrm{IR})$. The association between RA and IR, and its role on the different characteristics of the disease, such as duration and activity have not been well defined. In addition, there is a gap of knowledge regarding the link between systemic/local inflammation and insulin sensitivity and lipid metabolism in RA patients.

Objectives: To explore the effects of the inflammation on the glucose and lipid metabolism in the RA context, following three strategies: RA patients, collagen induced arthritis (CIA) mouse model and in vitro treatment of 3T3L1 adipocytes. Methods: Human study: 150 RA patients and 40 healthy donors were included. IR was quantified using the homeostatic model assessment of IR (HOMA-IR). Mouse model: $20 \mathrm{CB} 57 \mathrm{~J} / \mathrm{BL}$ mice were used; 5 mice were used as non-diseased group, and 15 were used in CIA modelling: sorted in low and high activity of the disease based on the number of inflamed digits depending on the duration of the disease. Plasma, leukocytes, skeletal muscle, liver and adipose tissue were collected. Treatment of adipocytes with serum from RA patients: 3T3L1 adipocytes were treated with serum $10 \%$ of RA patients and healthy donors for 24h. The expression of genes and proteins involved in inflammation, lipid metabolism and insulin signalling was analysed in all the tissues and cells.

Results: Percentages of obesity, hypertension, atherogenic risk, metabolic syndrome and insulin resistance were significantly increased in the RA group. Although mean time of evolution was 7 years, no association between IR and the duration of the disease was found. Levels of HOMA-IR significantly correlated with DAS28 and C-reactive protein levels, suggesting that systemic inflammation might lead to the development of insulin resistance. In mice, the induction of arthritis promoted an alteration of the expression of genes involved in inflammation as well as lipid metabolism and insulin signalling in all the metabolic tissues and leukocytes, pointing out to an increase in lipolysis, decrease in adipogenesis and lipid accumulation and induction of IR. These results were recapitulated after treatment of adipocytes with serum from RA patients with high disease activity. Conclusions: 1) IR was closely associated with an increase in disease activity and systemic inflammation in RA patients. 2) Induction of arthritis in mice promoted an increase in inflammation markers in skeletal muscle, adipose tissue and leukocytes, and a reduction of genes involved in lipid uptake and storage, generating an insulin resistance state in those tissues. 3) The inflammatory components in RA serum induced lypolisis, reduced adipogenesis, and increase inflammation and insulin resistance in adipocytes 3T3L1.

In sum, our results suggest that chronic inflammation associated with RA might directly impact relevant metabolic tissues, altering glucose and lipid homeostasis and favouring the development of insulin resistance.

Acknowledgements: Funded by ISCIII-FIS (CP15/00158), PI2013/0191, RD16/0012/0015 and Roche Pharma S.A.
Disclosure of Interest: None declared

DOI: 10.1136/annrheumdis-2017-eular.4725

\section{SATURDAY, 17 JUNE 2017 Trials and tribulations of medication adherence}

\section{OP0337-HPR PREDICTORS OF PATIENT REPORTED DECISION TO DISCONTINUE ANTI-RHEUMATIC MEDICATION IN RHEUMATOID ARTHRITIS PATIENTS: DATA FROM A RHEUMATOID ARTHRITIS COHORT}

V. Ahluwalia ${ }^{1}$, M. Movahedi ${ }^{2,3}$, E. Rampakakis ${ }^{3}$, A. Cesta ${ }^{2}$, X. $\mathrm{Li}^{2}$, S. Couto ${ }^{2}$ ${\mathrm{J} . ~ S a m p a l i \mathrm{~S}^{3}}$, C. Bombardier ${ }^{2,4,5}$ on behalf of OBRI investigators. ${ }^{1}$ Department of Rheumatology, Brampton Civic Hospital, William Osler Health System, Brampton; ${ }^{2}$ Ontario Best Practices Research Initiative, Toronto General Hospital, University Health Network, Toronto; ${ }^{3}$ JSS Medical Research, Montreal;

${ }^{4}$ Department of Medicine (DOM) and Institute of Health Policy, Management, and Evaluation (IHPME), University of Toronto; ${ }^{5}$ Division of Rheumatology, Mount Sinai Hospital, Toronto, Canada

Background: Despite the availability of safe and effective treatments and the establishment of treatment guidelines, real-world effectiveness remains suboptimal largely due to low patient adherence with prescribed treatment.

Objectives: The purpose of this study was to systematically evaluate sociodemographic, health insurance, and disease-related factors associated with patient reported decision for discontinuation of anti-rheumatic medications (ARM) in a large observational cohort of RA patients followed in Canadian routine clinical care.

Methods: RA patients enrolled in the Ontario Best Practices Research Initiative (OBRI) clinical registry and had at least two years of follow-up were included in the analysis. Treatment discontinuation due to patient reported decision was defined as ARM discontinuation. Independent predictors of ARM discontinuation were evaluated with multivariate cox-regression using both time-fixed and timedependent variables. Factors considered included patient sociodemographics (age, gender, race, education status, annual income, smoking history), health insurance information (private vs. non-private, \% coverage), disease parameters (RA duration, presence of erosion, RF positivity, DAS28, physician global, HAQ-DI, number of comorbidities), types of medications used, and physician characteristics (gender, academic position, urban vs. rural, distance from patient's residence)

Results: A total of 1,762 patients were included in the analysis with a mean (SD) age of 57.4 (13.0) years and disease duration of 8.5 (9.3) at the time of enrolment to the registry (baseline). The majorities of patients were female $(77.7 \%)$, had post-secondary education (55.3\%), and had private insurance $(67.2 \%)$. In terms of disease severity, $54.5 \%$ had prior erosion, $69.5 \%$ were RF positive, and mean (SD) DAS28 was 4.5 (1.5).

In a multivariate analysis, married status ( $\mathrm{HR}, 0.73 ; 95 \% \mathrm{Cl} 0.56-0.96)$, RF positivity ( $\mathrm{HR}, 0.73 ; 95 \% \mathrm{Cl} 0.56-0.96)$, and higher number of comorbidities ( $\mathrm{HR}, 0.92 ; 95 \% \mathrm{Cl} 0.85-0.99$ ) were identified as significant predictors of ARM continuation while higher physician global score (HR, 1.10; 95\% Cl 1.04-1.15), NSAID use (HR, 1.75; 95\% Cl 1.29-2.38), and polypharmacy (HR, 1.23; 95\% $\mathrm{Cl} 1.07-1.40$ ) were associated with ARM discontinuation due to patient reported decision.

Conclusions: In this systematic approach a variety of factors encompassing sociodemographics, disease, and medication characteristics, were identified as significant independent predictors of ARM discontinuation due to patient reported decision. These results should be taken into consideration when developing patient adherence support programs and in the choice of treatment regimens.

Disclosure of Interest: V. Ahluwalia Grant/research support from: OBRI was funded by peer reviewed grants from CIHR (Canadian Institute for Health Research), Ontario Ministry of Health and Long-Term Care (MOHLTC), Canadian Arthritis Network (CAN) and unrestricted grants from: Abbvie, Amgen, Bristol Myers Squibb, Celgene, Hospira, Janssen, Pfizer, Roche, Sanofi, \& UCB, M. Movahedi Employee of: OBRI/JSS, E. Rampakakis Employee of: JSS Medical Research, A. Cesta Employee of: OBRI, X. Li Employee of: OBRI, S. Couto Employee of: OBRI, J. Sampalis Employee of: Head of JSS, C. Bombardier Grant/research support from: OBRI was funded by peer reviewed grants from CIHR (Canadian Institute for Health Research), Ontario Ministry of Health and Long-Term Care (MOHLTC), Canadian Arthritis Network (CAN) and unrestricted grants from: Abbvie, Amgen, Bristol Myers Squibb, Celgene, Hospira, Janssen, Pfizer, Roche, Sanofi, \& UCB

DOI: 10.1136/annrheumdis-2017-eular.1522 